\title{
Le tinkering au cœur du processus de résolution de problèmes en contexte de fabrication numérique à l'école
}

$\begin{aligned} & \text { Auteurs Université de } \\ & \text { Caitlin Furlong, Uncton, Canada, } \\ & \text { caitlin.furlong@umoncton.ca }\end{aligned}$
$\begin{aligned} & \text { Michel T. Léger, Université de Moncton, Canada, } \\ & \text { michel.leger@umoncton.ca }\end{aligned}$




\section{REVUE HYBRIDE DE L'ÉDUCATION}

\section{Résumé}

À l'ère du $21^{\mathrm{e}}$ siècle, la littérature scientifique fait ressortir la résolution de problèmes comme l'une des compétences les plus recherchées par les employeurs à travers le monde. Cependant, peu d'écrits semblent s'être penchés sur le développement de cette compétence à l'école, surtout en contexte numérique. Au Nouveau-Brunswick, un changement de finalités de l'école implique davantage un enseignement axé sur les compétences, dont la résolution de problèmes. Par conséquent, appuyés par les écrits en technopédagogie, de nouveaux milieux d'apprentissage tels que les laboratoires de fabrication numérique peuvent représenter des environnements propices au développement de la résolution de problèmes. À partir d'un devis qualitatif interprétatif mené dans les laboratoires de fabrication numérique de deux écoles au Nouveau-Brunswick, l'analyse des résultats de la présente étude permet d'identifier le tinkering comme concept central dans les processus de résolution de problèmes. Notre recherche permet aussi de pointer vers d'autres éléments conceptuels importants entourant ce processus tel que l'étayage pédagogique et le degré d'autonomie des élèves. Enfin, notre étude confirme que la résolution de problèmes n'est pas un processus linéaire ; elle se déroule plutôt de façon itérative et peut être influencée par le niveau de compétences numériques des élèves.

Mots-clés : résolution de problèmes; laboratoires de fabrication; fabrication numérique; école; bricolage 


\section{$\overbrace{0}$ \\ REVUE HYBRIDE DE L'ÉDUCATION}

\section{Problématique}

Dans la société actuelle, les technologies font partie de plusieurs sphères de la vie, et ce, tant sur le plan personnel que professionnel. Le monde numérique dans lequel nous vivons fait en sorte que les compétences nécessaires pour réussir sur le marché du travail diffèrent de celles recherchées par le passé. Toutefois, pour bien s'adapter à cette nouvelle réalité, réussir sur le plan académique n'est pas suffisant (Gewertz, 2007). Spécifiquement, une des compétences jugées indispensables par les employeurs est la résolution de problèmes (Economist Intelligence Unit, 2015). Selon Poirier (2001), nous devons faire face à des situations problématiques afin d'apprendre. Depuis plusieurs années, la résolution de problèmes est un élément important des programmes d'études du Nouveau-Brunswick (N.-B.), de la maternelle à la douzième année (Ministère de l'Éducation et du Développement de la petite enfance [MEDPE], 2018). L'importance accordée à l'acquisition de cette compétence au N.-B. est observable dans les programmes d'études du Québec (Ministère de l'Éducation et de l'Enseignement supérieur [MEES], 2019) et même ailleurs au Canada. Cependant, selon les premiers résultats du Programme pour l'évaluation internationale des compétences des adultes (PEICA, 2012), les jeunes Canadiens démontrent toujours un certain manque de compétences quant à la résolution de problèmes, surtout dans des environnements riches en technologies (Statistique Canada, 2013). Comme le soulignent Hesse et ses collaborateurs (2015), il est intéressant de constater qu'être capable de résoudre des problèmes dans des environnements riches en technologie (ERT) est une habileté qui devient de plus en plus importante à l'ère numérique. En fait, nous nous demandons comment un milieu scolaire plutôt numérique, c'est-à-dire riche en technologies, peut possiblement influencer le développement de cette compétence de résolution de problèmes.

Maintenant le $21^{\text {e }}$ siècle bien entamé, Siddiq et ses collaborateurs (2017) nous rappellent que la place du numérique et la disponibilité d'une panoplie de technologies ne font que grandir dans toutes les sphères d'une société de plus en plus mondiale. Ces mêmes auteurs affirment que cette présence du numérique dans la société moderne entraîne de nombreux changements dans différents milieux de travail, notamment dans le domaine de l'éducation. L'émergence constante de nouvelles technologies nous donne l'occasion d'adopter une notre vision de l'éducation davantage alignée avec un paradigme favorisant l'innovation (Groff, 2013). Selon Schleicher (2018), si le système d'éducation ignore ce virage sociétal continu vers le numérique et n'adopte pas une posture de réforme active en ce sens, l'écart entre ce que l'école fournit et ce que la société exige va continuer d'augmenter. Plusieurs auteurs (Dede, 2010 ; Griffin et al., 2012 ; Siddiq et al., 2017) prétendent que les compétences du $21^{\mathrm{e}}$ siècle, notamment la résolution de problèmes, deviendront essentielles pour bien fonctionner dans un monde «connecté ». Or, force est de constater que 


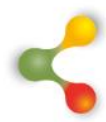

\section{REVUE HYBRIDE DE L'ÉDUCATION}

l'école traditionnelle demeure un milieu peu propice au développement de telles compétences (Groff, 2013).

Avec la croissance du maker movement ${ }^{1}$, nos communautés et nos écoles voient l'apparition d'environnements connus plus communément sous le nom de makerspaces ou Fab Labs (van Holm, 2014). Cross (2017) définit un makerspace comme "un espace où les étudiants créent des projets de passion autogérés et des prototypes d'inventions, et ils apprennent de nouvelles compétences en fonction de leurs intérêts, grâce à la collaboration et au bricolage » (p. 3). Selon Rosa et ses collaborateurs (2017), l'aspect numérique est une partie intégrale des Fab Labs, mais peut être absent dans les makerspaces. Au N.-B., ce type d'environnement est connu sous le nom de Labos Créatifs (ou Brilliant Labs en anglais). Pour leur part, ces Labos Créatifs sont décrits comme des laboratoires des makerspaces puisque les élèves ont la chance de travailler avec plus que seulement le numérique. Par exemple, certains de ces laboratoires sont équipés avec des machines pour faire de la couture (Leblanc et al., 2018). Cependant, notre étude se concentre seulement sur l'aspect numérique de ces laboratoires, donc nous utilisons le terme général laboratoire de fabrication numérique.

De nombreuses études semblent suggérer que les laboratoires de fabrication numérique peuvent favoriser le développement de la résolution de problèmes (Cross, 2017 ; Furlong et al., 2019; Peppler et al., 2015). Devant une croissance de ces espaces d'apprentissage dans les écoles du pays, particulièrement au N.-B. (LeBlanc et al., 2018), il semble pertinent d'étudier davantage comment les élèves résolvent des problèmes dans les laboratoires de fabrication numérique de leurs écoles. Parmi les études que nous avons recensées portant sur la résolution de problèmes dans ces environnements d'apprentissage, plusieurs auteurs affirment que le tinkering ${ }^{2}$ représente une façon particulièrement efficace de résoudre des problèmes (Poce et al., 2015 ; Vossoughi et Bevan, 2014). Par ailleurs, dans une étude antérieure menée au N.-B. sur le développement de compétences numériques dans les laboratoires de fabrication numérique (Furlong et al., 2019), nous avons remarqué la présence du tinkering chez un grand nombre des participants engagés dans un processus de résolution de problèmes. Dans cette même étude, il est intéressant de noter que d'autres problèmes sous-jacents survenaient quand les jeunes faisaient du tinkering. Bien que nous ayons observé le tinkering dans nos recherches sur la résolution de problèmes, tout comme Parkeh et Gee (2018), plusieurs questions demeurent quant à notre compréhension de la place du tinkering relativement aux mécanismes du processus de résolution de problèmes en laboratoire de fabrication numérique. En outre, en examinant les écrits en éducation et en technopédagogie sur le sujet en

\footnotetext{
1 « Une attitude d'ingéniosité qui a ses racines dans la culture du Do it yourself (DIY, bricolage,) 》 (Cross, 2017, p. 180).

2 Une disposition personnelle à résoudre des problèmes par l'investigation scientifique, l'expérimentation directe et la découverte (Martinez et Stager, 2013 ; Poce et al., 2019).
} 


\section{6}

\section{REVUE HYBRIDE DE L'ÉDUCATION}

contexte scolaire, notamment en contexte de laboratoire de fabrication numérique, nous constatons qu'il ne semble pas y avoir de données probantes qui explicitent la place du tinkering dans le développement de cette compétence. Ainsi, nous soulignons la pertinence de poursuivre les recherches sur le sujet afin de mieux informer les acteurs des systèmes scolaires partout au Canada, et ainsi, améliorer les stratégies pédagogiques de développement de résolution de problèmes chez les jeunes ${ }^{3}$. Plus précisément, dans le contexte des Labos Créatifs où les jeunes ont accès à l'internet pour faire des recherches et où les projets diffèrent d'une école à l'autre, nous nous demandons : quelle est la place du tinkering dans le processus de résolution de problèmes?

\section{Cadre conceptuel}

Avant d'aborder la résolution de problèmes dans les laboratoires de fabrication numérique, il importe de définir la résolution de problèmes. Selon l'Organisation de coopération et de développement économiques (OCDE, 2012), un problème est une situation où un obstacle ou défi nous empêche d'accomplir un but. La capacité de résoudre des problèmes est un processus cognitif complexe où l'individu doit s'apercevoir qu'il y a une différence entre l'état présent et l'état désiré pour atteindre un objectif, pour ensuite tenter d'agir sur la situation pour atteindre son objectif (Hesse et al., 2015 ; OCDE, 2013).

\section{Résolution de problèmes en environnement riche en technologie (ERT)}

L'arrivée des technologies amène une différente dimension à la résolution de problèmes. En fait, les technologies peuvent nous aider à résoudre des problèmes, mais peuvent aussi en engendrer, surtout si notre expérience avec les technologies utilisées est limitée (OCDE, 2012). Ainsi, I'OCDE (2012) propose une définition de la résolution de problèmes en ERT : «l'utilisation des technologies numériques, des outils de communication et des réseaux pour acquérir et évaluer des informations, communiquer avec les autres et effectuer des tâches pratiques »(p. 47). Toujours selon l'OCDE, il existe trois dimensions de résolution de problèmes en ERT; premièrement, l'identification du problème et des conditions nécessaires pour sa résolution; deuxièmement, la dimension cognitive, dont le processus mental pour résoudre le problème; et troisièmement, les technologies, soit les outils qui permettent la résolution de problèmes.

II existe principalement trois types de problèmes auxquels nous pouvons faire face dans des ERT : un problème où la technologie est la

\footnotetext{
${ }^{3}$ Notre étude s'inscrit à un projet de recherche plus large mené par le réseau CompéTI.CA (partenariat stratégique pour comprendre l'écosystème, l'adaptabilité et le transfert de compétences numériques).
} 


\section{$\&$ \\ REVUE HYBRIDE DE L'ÉDUCATION}

cause du problème; un problème où la solution de ce dernier nécessite l'utilisation des technologies ; un problème qui est associé à la manipulation d'outils numériques (par exemple : la construction d'une imprimante 3D) (OCDE, 2012). Dans le contexte des ERT du laboratoire de fabrication numérique, il y a aussi deux distinctions à faire concernant la résolution de problèmes. En fait, celle-ci peut se présenter sous format d'un problème de grande envergure à résoudre comme objectif central. Par exemple, dans l'étude de Smith et al. (2015), le but des projets des élèves dans les laboratoires de fabrication numérique était de cibler un problème global qui affecte la société et de déterminer comment le résoudre avec l'aide des technologies. La résolution de problèmes peut aussi se présenter sous forme de processus ; les élèves font face à des problèmes qu'ils devront résoudre tout au long de la réalisation d'un projet. Dans le cas de notre étude, nous nous intéressons à cette deuxième forme de résolution de problèmes. Enfin, il semble y avoir une lacune au niveau de l'évaluation de la résolution de problèmes (Voogt et Roblin, 2012). De plus, certains affirment qu'il y a un manque d'instruments permettant d'évaluer cette compétence (Siddiq et al., 2016 ; Wilson et al., 2015). Finalement, bien qu'il existe des modèles de résolution de problèmes dans les ERT, tels que les modèles de Gauthier (2014) et de Kim et Hannafin (2010), les recherches ne semblent pas démontrer l'application de ces modèles au contexte des laboratoires de fabrication numérique. Le besoin d'approfondissement sur le sujet apparait essentiel.

\section{Le tinkering}

Le tinkering est un terme récurrent lorsque nous parlons du maker movement et des laboratoires de fabrication numérique (Poce et al., 2019). Resnick et Rosenbaum (2013) définissent le tinkering comme une sorte de fabrication qui met l'accent sur l'improvisation, l'exploration et l'expérimentation, de façon itérative, face à un problème ou à un projet. Au cœur du processus du tinkering se retrouvent le processus d'essai-erreur, entre autres, où les jeunes expérimentent, testent des idées, font des ajustements et retestent de façon continue (Resnick et Rosenbaum, 2013). Quand quelqu'un fait du tinkering, il " pense avec ses mains » (Wilkinson et Petrich, 2014, p. 13), il ne suit pas de règles ni d'objectifs prédéterminés ; il persiste pour essayer de trouver des solutions aux problèmes (Harris et al., 2016). Pour sa part, Doorley (2015) explique le tinkering comme un processus en soi qui permet l'apprentissage par l'échec. Selon Parkeh et Gee (2018), il n'y a pas de distinction claire entre la fabrication et le tinkering. De leur côté, Martinez et Stager (2013) différencient la fabrication du tinkering en expliquant qu'un jeune qui fabrique à souvent un produit final en tête, tandis qu'un jeune qui fait du tinkering essaie différentes choses et se laisse guider par ses résultats. Le tinkering peut aussi être compris comme le contraire de la planification (Resnick et Rosenbaum, 2013). Cependant, selon notre compréhension des écrits scientifiques sur le sujet, bien que quelqu'un qui tinker peut avoir un objectif en tête, voire un véritable plan, ce plan peut changer en cours de route face à son 


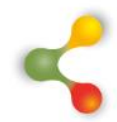

\section{REVUE HYBRIDE DE L'ÉDUCATION}

interaction avec les différents matériaux manipulés. Selon plusieurs auteurs, le tinkering s'explique par l'utilisation d'outils technologiques (circuits, robotique, impression 4D, arduino, etc.) et de matériaux communs, comme le plastique et le carton, pour créer des projets et non seulement pour l'assemblage (Bevan et al., 2015 ; Vossoughi et Bevan, 2014 ; Vossoughi et al., 2016,). De plus, le tinkering n'est pas seulement associé à une construction physique, mais peut aussi s'appliquer à une fabrication de nature virtuelle, en création d'un jeu électronique (Resnick et Rosenbaum, 2013).

Plusieurs auteurs tels que Petrich et ses collaborateurs (2013), Resnick et Rosenbaum (2013) ainsi que Bevan et ses collaborateurs. (2015) préfèrent utiliser l'expression activité de tinkering. D'après Bevan et al. (2015), l'essentiel d'une activité de tinkering est de « développer une idée personnellement significative, rester coincé dans certains aspects de la réalisation physique de l'idée, persister tout au long du processus et faire des percées lorsque l'on trouve des solutions aux problèmes » (p. 99). Pour eux, au cours d'une activité de tinkering, les apprenants créent quelque chose de nouveau de façon itérative, en utilisant des matériaux et des outils (Harris et al., 2016). De plus, les apprenants fixent leurs propres objectifs, formulent des hypothèses, se posent des questions, articulent les problèmes qu'ils rencontrent, testent leurs idées de façon improvisée et ludique, et modifient ou redéfinissent leurs objectifs de départs (Harris et al., 2016 ; Resnick et Rosenbaum, 2013). Pour Harris et ses collaborateurs (2016) spécifiquement, une activité de tinkering permet justement à l'élève de poser des problèmes à résoudre, d'identifier les problèmes émergents, de faire des remue-méninges et de trouver de nouvelles solutions face à des problèmes. En effet, dans une activité de tinkering, l'élève va plutôt créer quelque chose de nouveau. II ne fait pas simplement suivre des étapes d'un manuel, il planifie plutôt la réalisation de son projet en manipulant le matériel disponible de façon improvisée. Malgré les divergences des points de vue en ce qui a trait à la définition du tinkering, plusieurs auteurs semblent en accord avec le fait que le tinkering est une branche de la fabrication qui met l'accent sur l'improvisation et la résolution de problèmes en bricolant (Bevan et al., 2014 ; Bevan et al., 2015 ; Harris et al., 2016 ; Resnick et Rosenbaum, 2013). Nous considérons ainsi qu'une activité de tinkering en est une où l'apprenant fait du tinkering tout au long du processus de création du produit visé.

\section{Méthodologie}

Notre étude se situe dans l'approche qualitative interprétative où "le sens attribué à la réalité est vu comme étant construit entre le chercheur, les participants à l'étude et même les utilisateurs des résultats de la recherche » (Savoie-Zajc, 2018, p. 195). La pertinence de ce devis en éducation repose sur le fait qu'il soit fondé sur la création des relations entre la personne enseignante et les élèves. De plus, puisque le contexte oriente les résultats qui évoluent de façon continue (Savoie-Zajc, 2018), ce devis 


\section{6}

\section{REVUE HYBRIDE DE L'ÉDUCATION}

est pertinent pour notre recherche, car nous voulons étudier la réalité des élèves, les observer et interagir avec eux, afin de comprendre comment ils résolvent des problèmes dans le contexte précis des Labos Créatifs, dans les écoles francophones et anglophones. Le contexte est également important ici puisque les Labos peuvent prendre différentes formes, et ce, dans différentes provinces, et même, dans différentes écoles.

Les étapes de la recherche qualitative interprétative que nous avons suivies: la formulation d'une question de recherche, l'échantillonnage théorique, la collecte des données et l'analyse inductive des données. Nous avons continué les deux dernières étapes de façon cyclique jusqu'à la saturation des données.

\section{Collecte de données}

Dans une recherche qualitative interprétative, le choix des participants est intentionnel. D'après des critères établis à partir du cadre théorique, le chercheur choisit des participants qui ont certaines caractéristiques communes. Selon Lecompte et Preissle (1993), l'échantillon peut être formé à partir de groupes qui existent déjà ou des groupes formés par le chercheur. Dans notre étude, nous avons choisi des classes d'élèves qui utilisent le Labo Créatif de leur école pour faire des projets. Toutefois, nous avons choisi d'observer seulement certains élèves, soit ceux qui réalisaient leur projet avec le numérique. II existe plusieurs types d'échantillonnage intentionnel, tel que l'échantillonnage théorique faire une première sélection de participants avec la possibilité d'inclure ou d'enlever des participants ; plus la recherche avance, plus les compétences recherchées dans l'échantillon sont spécifiques (Savoie-Zajc, 2018). Ainsi, pendant l'année 2018-2019, nous avons choisi d'observer les élèves d'une classe de $5^{\mathrm{e}}$ année d'une école francophone ainsi qu'une classe de $11^{\mathrm{e} / 12^{\mathrm{e}}}$ année d'une école anglophone, travaillant sur des projets dans le Labo Créatif. Deux visites par école ont eu lieu afin de déterminer la présence de tinkering dans le processus de résolution de problèmes dans les laboratoires de fabrication numérique au primaire ainsi qu'au secondaire.

En recherche qualitative interprétative, nous retrouvons notamment trois modes de collectes de données : l'entrevue, l'observation et l'usage de matériel écrits (Savoie-Zajc, 2018). Selon l'auteure, l'observation ouverte et participante est importante en recherche qualitative interprétative. En cohérence, nous avons décidé de filmer les élèves pour pouvoir observer comment ils résolvent des problèmes, et au lieu d'utiliser des grilles d'observation, nous les avons questionnés sur la nature de leur projet et leur processus de résolution de problèmes. Notre questionnement était général nous permettant de mieux comprendre ce que faisaient concrètement les élèves : que faites-vous? Pourquoi faites-vous cela? Comment avez résolu ce problème ? Pouvez-vous me montrer? 


\section{$\&$}

\section{REVUE HYBRIDE DE L'ÉDUCATION}

À la suite de notre collecte de données, plusieurs questions sont survenues: quelles sont toutes les étapes encourues par l'élève pour résoudre un problème? Pourquoi certains élèves font-ils du tinkering en premier et d'autres non? Nous avions donc besoin d'avoir des renseignements plus pointus nous permettant de mieux comprendre la place du tinkering dans le processus de résolution de problèmes. En respectant la structure de l'échantillonnage théorique qui permet la modification de l'échantillon (Savoie-Zajc, 2018), nous avons décidé de retourner sur le terrain pendant l'année 2019-2020 afin d'effectuer des entretiens semi-dirigés. L'entretien semi-dirigé est recommandé pour assurer une constance entre les différents entretiens (Savoie-Zajc, 2018). Impossible de faire les entretiens avec les mêmes élèves, mais désireux de maintenir une certaine continuité entre les données (tableau 1), nous avons ciblé différents élèves, mais avec les mêmes personnes enseignantes. Les entretiens furent réalisés sur une base volontaire et avec des groupes d'élèves travaillant sur un projet ensemble. En fait, les entretiens nous ont permis de recueillir des renseignements sur les types d'élèves dans la salle de classe. La perspective enseignante était aussi pertinente puisqu'ils pouvaient refléter leurs expériences dans les laboratoires de fabrication des années précédentes, ainsi que de vulgariser la façon dont les élèves résolvent des problèmes. Finalement, les entretiens avec les élèves et les enseignantes combinés à nos observations permettent une triangulation des données, et ainsi, une meilleure crédibilité des résultats (Laperrière, 1997). Tout comme lors de notre première collecte de données, les élèves ciblés ne travaillaient que sur des projets en lien avec la technologie.

Tableau 1 : Profil des participantes et participants

\begin{tabular}{|c|c|c|c|}
\hline École & $\begin{array}{l}\text { Visites au } \\
\text { laboratoire }\end{array}$ & Année & Activités observées \\
\hline \multirow[t]{2}{*}{$\begin{array}{l}\text { Francophone } \\
5^{\mathrm{e}} \text { année }\end{array}$} & \multirow[t]{2}{*}{$\begin{array}{l}1-2 \text { fois } \\
\text { par semaine }\end{array}$} & $\begin{array}{l}2018- \\
2019\end{array}$ & $\begin{array}{l}\text { Impression 3D, programmation de moteurs, } \\
\text { robotique, vidéos accélérées de la croissance } \\
\text { des plantes }\end{array}$ \\
\hline & & $\begin{array}{l}2019- \\
2020\end{array}$ & $\begin{array}{l}\text { Bateaux et voitures électriques, ville faite de } \\
\text { matériaux recyclés, transformation d'un } \\
\text { aquarium en observatoire d'abeilles }\end{array}$ \\
\hline \multirow[t]{2}{*}{$\begin{array}{l}\text { Anglophone } \\
11^{\mathrm{e}}-12^{\mathrm{e}} \text { année }\end{array}$} & Chaque jour & $\begin{array}{l}2018- \\
2019\end{array}$ & $\begin{array}{l}\text { Robotique, sérigraphie, impression 3D de } \\
\text { pièces pour un moteur, vêtements } \\
\text { personnalisés avec un coupe-vinyle }\end{array}$ \\
\hline & $\begin{array}{l}2 \text { fois par } \\
\text { jour }\end{array}$ & $\begin{array}{l}2019- \\
2020\end{array}$ & $\begin{array}{l}\text { Robotique, sérigraphie, coupe-vinyle, } \\
\text { création de boutons, impression 3D }\end{array}$ \\
\hline
\end{tabular}




\section{6}

\section{REVUE HYBRIDE DE L'ÉDUCATION}

\section{Cadre d'analyse}

Pour donner suite à notre première phase de collecte de données, nous avons fait ressortir : les propos des jeunes pendant la résolution de problèmes en précisant le type de projet, nos observations en lien avec la résolution de problèmes, les citations des jeunes et les thèmes émergent de nos observations, et des citations. Nous avons aussi inclus des images des projets pour mieux comprendre la nature du celui-ci. Cette méthode d'analyse est inspirée par l'étude de Parkeh et Gee (2018) et de Bevan et al. (2015). Pour faire ressortir les thèmes, nous n'avons pas utilisé notre cadre théorique. Nous avons plutôt réalisé une analyse logique inductive modérée, c'est-à-dire que le cadre de référence utilisé pour définir le concept est mis de côté pour laisser émerger des catégories (Savoie-Zajc, 2000).

Pour notre deuxième ronde de collecte de données, nous avons effectué une analyse thématique des propos de l'entretien semi-dirigé en suivant encore une fois une logique inductive modérée. L'analyse thématique consiste à "procéder systématiquement au repérage, au regroupement et, subsidiairement, à l'examen discursif des thèmes abordés dans un corpus » (Paillé et Mucchielli, 2008, p. 162). Ainsi, nos verbatims furent transcrits et regroupés dans un tableau. Pour chaque énoncé, nous avons fait ressortir des thèmes et sous-thèmes.

\section{Résultats et discussion}

\section{Première collecte de données}

Suite à notre première analyse, nous remarquons tout d'abord que, quelle que soit la nature du projet, des éléments du tinkering sont présents dans la démarche de résolution de problèmes des élèves. Entre autres, les élèves résolvent des problèmes par le bricolage, ce qui est un élément de la définition du tinkering telle que ressortie par plusieurs auteurs (Bevan et al., 2014 ; Resnick et Rosenbaum, 2013).

Nous remarquons aussi que les élèves résolvent des problèmes de façon improvisée (Bevan et al., 2014 ; Resnick et Rosenbaum, 2013), par l'expérimentation directe (Martinez et Stager, 2013; Resnick et Rosenbaum, 2013) et en testant et retestant diverses idées (Harris et al., 2016; Resnick et Rosenbaum, 2013). Ce qui apparait comme étant particulièrement intéressant repose sur le fait que les élèves ne font pas simplement du tinkering pour résoudre leurs problèmes, ils débutent par l'identification du problème (Kim et Hannafin, 2010 ; OCDE, 2012), ils utilisent d'autres ressources technologiques, telles que l'internet, pour rechercher des pistes de solution (Gauthier, 2014 ; Kim et Hannafin, 2010) 


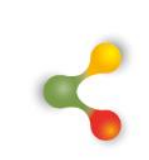

\section{REVUE HYBRIDE DE L’ÉDUCATION}

où de demander pour de l'aide à la personne enseignante ou des experts du domaine. Nous remarquons également que le processus de résolution de problèmes semble différer d'une école à l'autre, et même, d'un projet à l'autre.

Si nous prenons l'exemple d'un projet de l'école primaire francophone présenté (tableau 2), les élèves ont décidé de construire un système aquaponique, permettant de faire pousser des plantes à l'aide d'excrément de poisson et de nourrir les poissons à l'aide de plantes. Pendant la collecte de données, les élèves étaient à l'étape de construction de verres en plastique - pour mettre les plantes dedans - avec l'imprimante 3D. Les premières versions des verres n'ayant pas bien imprimé, les élèves ont dû ajuster les paramètres de l'application, et modifier le verre. Cet exemple représente bien le tinkering. Toutefois, les élèves ont témoigné que, lorsqu'ils ne sont pas capables de résoudre le problème par eux-mêmes, ils recherchent parfois en ligne et que, si ça ne fonctionne toujours pas, ils demandent à l'agent technopédagogique présent à l'école de temps à autre. 


\section{REVUE HYBRIDE DE L'ÉDUCATION}

Tableau 2 : Exemple d'observations des élèves francophones

\begin{tabular}{|c|c|c|c|}
\hline Projets & $\begin{array}{l}\text { Ce qu'on } \\
\text { observe }\end{array}$ & $\begin{array}{l}\text { Citation des élèves au niveau de } \\
\text { la résolution de problèmes (RP) }\end{array}$ & $\begin{array}{l}\text { Éléments de la RP } \\
\text { observés }\end{array}$ \\
\hline $\begin{array}{l}\text { Construction d'un système } \\
\text { hydroponique et aquaponique } \\
\text { pour faire pousser les plantes et } \\
\text { nourrir les poissons }\end{array}$ & $\begin{array}{l}\text { Les premières } \\
\text { versions ont } \\
\text { moins bien } \\
\text { imprimé, donc } \\
\text { ils ont ajusté } \\
\text { les paramètres } \\
\text { et ont } \\
\text { recommencé. } \\
\text { Les élèves } \\
\text { font des } \\
\text { recherches } \\
\text { pour voir } \\
\text { comment le } \\
\text { diminuer le } \\
\text { bruit de } \\
\text { l'imprimante }\end{array}$ & $\begin{array}{l}\text { C4 : Donc quand vous avez des } \\
\text { problèmes, vous essayez de le } \\
\text { résoudre vous-mêmes? } \\
\text { EF25 : On ne demande pas à la } \\
\text { madame. On fait des recherches } \\
\text { sur l'ordinateur pour qui se vire... } \\
\text { comme maintenant ça fait du bruit, } \\
\text { puis maintenant on est en train de } \\
\text { faire des recherches pour trouver } \\
\text { comment arrêter le bruit... } \\
\text { EF2: La madame ne sait pas } \\
\text { [comment ça fonctionne], on a } \\
\text { développé comme une pensée } \\
\text { critique, puis comme on se } \\
\text { demande... quand on veut } \\
\text { commencer on se demande tout le } \\
\text { temps une question : est-ce que ça } \\
\text { va marcher, puis est-ce que ça fait } \\
\text { du sens? } \\
\text { EF1: Ça nous sert comme à être } \\
\text { plus autonome parce qu'au début } \\
\text { de l'année, on était toujours comme } \\
\text { madame on peut faire ça? } \\
\text { Maintenant, on fait les choses } \\
\text { comme pas mal toujours tout seul. } \\
\text { EF2 : Eux deux ont commencé à } \\
\text { construire l'imprimante... On a } \\
\text { toute fait des recherches puis on a } \\
\text { comme trouvé comment ça } \\
\text { marchait. On a fixé comme des } \\
\text { problèmes. Comme le bec, ben il } \\
\text { marchait pu. En premier on voulait } \\
\text { juste explorer, puis là, après là, on } \\
\text { a fait des recherches. Finalement, } \\
\text { c'est à cause que ça ne tournait } \\
\text { pas. Mais c'était juste brisé. On } \\
\text { avait tout essayé sauf ça puis le }\end{array}$ & 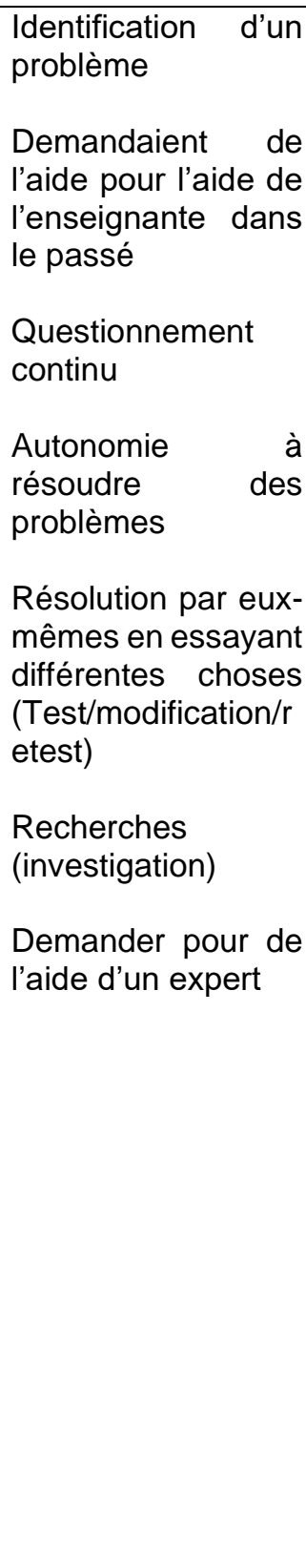 \\
\hline
\end{tabular}

${ }^{4} \mathrm{C}$ : Chercheuse

${ }^{5} \mathrm{E}$ : Élève de l'école francophone 


\section{$\&$}

\section{REVUE HYBRIDE DE L'ÉDUCATION}

\begin{tabular}{|l|l|}
\hline & $\begin{array}{l}\text { monsieur est arrivé, puis dans } \\
5 \text { minutes il a trouvé que c'était ça. } \\
\text { E2 : Ben, on avait un cube qui était } \\
\text { tout cassé, donc notre deuxième } \\
\text { print fallait qu'on pèse nous- } \\
\text { mêmes, puis ça pris comme 1h30. } \\
\text { Ça ne fonctionnait pas bien parce } \\
\text { que le filament ne rentrait pas } \\
\text { dedans le petit moteur ici. Là, le } \\
\text { monsieur est venu puis il a dit c'est } \\
\text { à cause que le filament ne rentrait } \\
\text { pas donc il l'a arrangé. } \\
\text { E1: Il y a des fois où ça ne marche } \\
\text { pas trop bien, donc on le modifie } \\
\text { juste un petit peu. }\end{array}$ \\
\hline
\end{tabular}

Un exemple provenant de l'école anglophone secondaire : l'élève a décidé de faire de la sérigraphie pour mettre un logo sur des chandails dans le but de créer sa propre compagnie. Avant de débuter, il a regardé sur YouTube pour savoir quoi faire et pour éviter des problèmes. Par contre, en amorçant le projet, il a remarqué qu'il n'avait pas d'émulsion à photo, donc il devait improviser pour transférer le logo (faire du tinkering). II a alors décidé d'utiliser du vinyle. II a testé son idée, ce qui n'a pas fonctionné. L'enseignant est ensuite venu vers lui en le questionnant à propos du processus. Ils ont alors fait un remue-méninge ensemble pour trouver une idée (Harris et al., 2016) : mettre plus de pression. Ici, l'élève a fait du tinkering et l'enseignant a agi à titre de facilitateur durant le processus (une des étapes de la résolution de problèmes dans les salles de classe riches en technologies, selon Kim et Hannafin [2010]). L'élève a retesté cette nouvelle idée qui n'a pas fonctionné. Resnick et Rosenbaum (2013) affirment que plusieurs éducateurs pensent qu'en faisant du tinkering, les jeunes ne vont pas comprendre ce qu'ils font. Nous croyons toutefois que dans plusieurs cas, cette expérimentation leur permet au contraire de développer leur propre compréhension (Hancock, 2003). Ici, l'élève a pu expliquer pourquoi le processus initial n'a pas fonctionné et pourquoi cela ne fonctionnera pas avec le vinyle. La question suivante se pose : si le projet n'avait pas été un projet innovateur et que l'élève avait pu trouver la réponse sur l'internet, est-ce qu'il aurait fait du tinkering?

De façon générale, nous avons remarqué que le tinkering est présent dans le processus de résolution de problème. Toutefois, nous avons aussi remarqué que les élèves utilisaient aussi plusieurs autres façons de résoudre des problèmes, soit en faisant de l'investigation par le biais des recherches sur l'internet, en suivant les étapes d'un guide ou d'un tutoriel, ou en demandant à un enseignant. De plus, l'ordre des étapes semblait différer d'un projet à l'autre et avec seulement deux visites par 


\section{$\&$}

\section{REVUE HYBRIDE DE L'ÉDUCATION}

école, il est apparu difficile de déterminer le rôle du tinkering dans ce processus. Est-ce que les jeunes passent la majorité du temps à faire du tinkering? Est-ce qu'ils font du tinkering seulement quand ils ne peuvent pas trouver les réponses « ailleurs »?

\section{Deuxième collecte de données}

Suite à cette deuxième ronde de collecte de données, nous avons remarqué que la place que le tinkering occupe dans le processus de résolution de problèmes différait d'un élève à l'autre, d'une école à l'autre et d'un projet à l'autre. Dans plusieurs cas, les élèves demandent de l'aide à la personne enseignante aussitôt qu'ils ont un problème. Dans le cas des groupes observés, les personnes enseignantes les dirigeaient vers la recherche ou du tinkering. Certains élèves se servaient seulement du tinkering pour résoudre leurs problèmes, tandis que d'autres faisaient seulement du tinkering s'ils ne pouvaient pas trouver la réponse en faisant de la recherche. Dans d'autres cas, les élèves débutaient en faisant du tinkering et de la recherche, et lorsqu'ils ne pouvaient pas trouver la réponse, ils demandaient de l'aide.

Dans les Labos Créatifs, la majorité des projets sont innovants ce qui rend difficile de trouver les réponses à leurs problèmes en ligne. Les élèves doivent donc faire du tinkering à un moment ou à un autre :

$\mathrm{A}^{6}$ : ils essaient une chose et ils comprenaient que ce n'est pas ça et ils essayaient une autre chose (traduction libre).

Un constat intéressant est que dans la plupart des cas, même si les élèves pouvaient trouver la réponse à leur problème en ligne, ils commençaient en faisant du tinkering, et ce, même si leur enseignant leur déconseillait.

A : quand les élèves ont un problème et on essaie de les entraîner à ne pas faire ça, mais... ils essaient de faire du tinkering pour résoudre le problème en utilisant l'équipement, et ce que j'essaie de leur apprendre à faire, c'est de la recherche, car ce problème aurait pu avant à quelqu'un (traduction libre).

Ainsi, l'enseignant essaie de les encourager à faire de la recherche avant et s'ils ne peuvent pas trouver la réponse à leur problème, de faire du tinkering. Par contre, la plupart du temps, ce n'est pas ce que les élèves choisissent de faire. Selon l'enseignant, quand l'élève choisit le tinkering pour résoudre un problème, il va souvent engendrer d'autres problèmes, ce qui favorise le découragement. Selon l'enseignant, le tinkering peut aussi ruiner le matériel. Par exemple, dans le cas d'un problème avec le

\footnotetext{
${ }^{6}$ Enseignant de l'école anglophone
} 


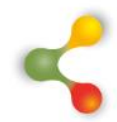

\section{REVUE HYBRIDE DE L'ÉDUCATION}

fonctionnement ou l'assemblage d'une imprimante 3D, la réponse au problème peut se trouver en ligne et en faisant du tinkering. Dans ce cas, cela pourrait abimer l'imprimante. Martinez et Stager (2013) font la même remarque en disant qu'il y a des circonstances où il est mieux de ne pas faire du tinkering; quand le risque d'erreur peut être dangereux ou coûteux, il apparait préférable d'explorer différentes options.

\section{Facteurs d'influence}

Dans les deux écoles, les groupes d'élèves de l'année 2018-2019 étaient différents des élèves de l'année 2019-2020. Dans le cas de l'école francophone, l'enseignante avait principalement des élèves qui n'avaient aucune expérience dans le Labo Créatif. Dans l'école anglophone, les élèves de l'année 2018-2019 avaient le cours de Labo Créatif combiné avec le cours d'entrepreneuriat, donc les élèves créaient des produits qu'ils voulaient vendre, ce qui n'était pas le cas pour les élèves de l'année suivante. Ainsi, nos données provenaient de groupes différents de l'année précédente, ce qui peut expliquer les différences observées.

Plusieurs facteurs qui peuvent influencer la place que le tinkering occupe dans la démarche de résolution de problèmes. En voici sept :

1) Niveau d'habileté des enseignantes et enseignants

Dans l'école anglophone, l'enseignant était très habile avec les technologies : plusieurs élèves avaient tendance à aller voir l'enseignant pour de l'aide pour des problèmes auxquels ils auraient pu trouver la réponse en ligne. Les élèves faisaient soit un peu de tinkering et s'ils ne pouvaient pas trouver la réponse, ils allaient voir l'enseignant, ou ils allaient directement le voir. Dans le cas de l'école francophone, en 2018-2019, on voyait surtout les élèves résoudre les problèmes par eux-mêmes en faisant du tinkering ou des recherches. Pour le groupe d'élèves 2017-2018, pour les élèves qui avaient des projets moins complexes, ils avaient tendance à demander à l'enseignante immédiatement, mais pas dans le cas de projets plus complexes: «la madame ne saurait pas comment ça marche » et avaient plus tendance à résoudre les problèmes par eux-mêmes.

\section{2) L'expérience dans le laboratoire}

Dans les deux écoles, les élèves qui avaient déjà travaillé dans un Labo Créatif étaient plus à l'aise de résoudre un problème par eux-mêmes. Si nous prenons l'exemple du groupe du primaire de l'année 2019-2020 où la majorité des jeunes n'étaient pas autonomes et n'avaient pas travaillé dans un Labo Créatif avant, l'enseignante pouvait déjà remarquer une grosse différence sur le plan de l'autonomie des élèves pour résoudre des problèmes en janvier, comparativement à septembre. 


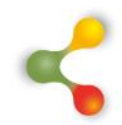

\section{REVUE HYBRIDE DE L'ÉDUCATION}

$\mathrm{F}^{7}$ : Ils ont tendance à venir directement pour moi, puis c'est ça que je trouve difficile parce que l'année passée, les élèves avaient compris le message beaucoup plus tôt dans l'année; ils avaient compris OK, si j'ai une question-là, je vais essayer d'y répondre en utilisant mes stratégies avant d'aller mon enseignante.

\section{3) Compétences numériques des élèves}

Les élèves qui avaient une facilité avec les technologies avaient tendance à ne pas demander de l'aide à la personne enseignante et pouvaient souvent régler le problème par eux-mêmes en faisant du tinkering ou de la recherche. Ces élèves avaient aussi tendance à faire des projets plus complexes et innovants qui nécessitaient le tinkering.

4) Complexité du projet

Les élèves qui ont fait face à des projets plus complexes ont dû résoudre plus de problèmes.

$\mathrm{F}:$...si on fait référence justement au projet des papillons là, eux ils ont constamment des problèmes puis ils ont la chance de les résoudre, puis ils les résoudent vraiment bien... puis les autres c'est parce que leur projet n'est pas assez complexe, c'est une ligne. II n'y a pas d'embuche ou rien.

L'enseignante de l'école primaire affirme que les élèves qui choisissent des projets complexes sont davantage capables de les résoudre de façon autonome.

$\mathrm{F}$ : les élèves qui ont choisi les plus complexes sont mieux pour ça. Ils vont essayer de trouver des solutions parce que... ben, je trouve que c'est dans leur nature.

D'après Blikstein (2013), les élèves tombent souvent dans le piège, appeler le syndrome du porte-clés, où ils produisent quelque chose de simple et étant encouragé par la réussite de cette production. Ils produisent plusieurs versions du produit et le laboratoire de fabrication devient ainsi une usine de fabrication au lieu d'un endroit où innover. En reproduisant la même chose à plusieurs reprises, les problèmes vont disparaître. Ce processus a pu être observé chez les groupes de l'école anglophone qui ont produit des dizaines de chandails avec des logos.

\footnotetext{
${ }^{7}$ Enseignante de l'école francophone
} 


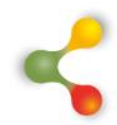

\section{REVUE HYBRIDE DE L'ÉDUCATION}

\section{5) Degré d'innovation du projet}

Plus un projet est innovant, plus les élèves devront faire du tinkering pour trouver la solution à leurs problèmes, car si le projet n'a jamais été fait avant, il y a peu de chances qu'ils trouvent leur réponse sur l'internet. Pour ce genre de projets, si un élève est moins motivé, a moins d'intérêt ou s'il est habitué à recevoir une solution rapidement, il aura davantage tendance à abandonner. Ce qui nous amène aux deux prochains facteurs.

6) Niveau d'intérêt ou de motivation envers le projet

Comme mentionné par Harris et ses collaborateurs (2016), dans une activité de tinkering, les élèves doivent avoir un intérêt pour le projet. Dans notre étude, nous avons remarqué que chez les élèves qui ont fait un peu de tinkering et qui ont ensuite abandonné, plusieurs d'entre eux n'avaient pas d'idées pour le projet et ont choisi quelque chose qui avait l'air intéressant. En conséquence, lorsqu'ils ont vu les autres faire, et lorsque la résolution de problème est devenue trop difficile, les élèves ont abandonné. D'autre part, certains élèves moins motivés choisissent des projets très simples et non innovants, ce qui fait en sorte qu'il y a rarement de problèmes et s'il y en a, ils peuvent facilement être résolus en regardant sur l'internet. Les élèves moins motivés ont tendance à demander plus d'aide aux personnes enseignantes, qui agissent comme des facilitateurs les dirigent vers des ressources en ligne ou du tinkering.

\section{7) Expérience à résoudre des problèmes}

Un enseignant a répondu que la façon dont les élèves ont appris à résoudre des problèmes peut être un facteur.

A : S'ils ont suivi des cours où ils devaient essentiellement résoudre des problèmes seuls, ils essaieront souvent de faire de leur mieux pour comprendre ce qui ne va pas (traduction libre).

Ainsi, si les élèves sont habitués à toujours recevoir la réponse rapidement, ils vont avoir tendance à demander pour de l'aide ou à se décourager plus rapidement s'ils ne trouvent pas la réponse en faisant du tinkering.

\section{Conclusion}

Le tinkering apparait essentiel dans le monde dans lequel nous vivons notamment en raison des changements rapides, de l'adaptation nécessaire ainsi que de la recherche de solutions créatives et innovatrices à de nouveaux problèmes qui peuvent survenir (Resnick et Rosenbaum, 2013). Dans le cadre de cette étude, nous avons déterminé que le tinkering 


\section{$\&$}

\section{REVUE HYBRIDE DE L'ÉDUCATION}

est présent dans la démarche de résolution de problèmes dans les laboratoires de fabrication numérique, où les élèves testent et retestent leurs idées de façon improvisée par l'expérimentation directe (Harris et al., 2016 ; Martinez et Stager, 2013 ; Resnick et Rosenbaum, 2013). Ce que nous remarquons dans les Labos Créatifs : même si les élèves ont accès à l'internet pour trouver des solutions à leurs projets et que ce ne sont pas tous les projets qui nécessitent du tinkering, pour plusieurs élèves, faire de la recherche en ligne n'est pas nécessairement leur premier réflexe. Pour plusieurs projets, les élèves font du tinkering avant de faire une recherche sur l'internet. De plus, nous remarquons que, peu importe le projet ou l'école, le tinkering occupe une place importante dans le processus de résolution de problèmes et que ce processus dépend de plusieurs facteurs. Cependant, l'ordre, la place et l'importance qui est accordée à chaque étape dépendent de plusieurs facteurs, tels que les compétences numériques de la personne enseignante et des élèves, le degré d'expérience des élèves dans le laboratoire, le degré d'innovation et de complexité du projet, l'habileté académique des jeunes ainsi que le niveau de motivation des jeunes.

Dans cette étude, nous avons remarqué la présence non seulement du tinkering dans le processus de résolution de problèmes, mais aussi d'autres composantes telles que l'identification du problème, l'investigation sur l'internet, la demande d'aide et le rôle de la personne enseignante comme facilitateur. Nous avons aussi perçu des similitudes entre ces composantes et certaines qui se retrouvent dans les modèles de résolution de problèmes de Gauthier (2014) et Kim et Hannafin (2010). Ainsi, comme prochaine étape de ce projet, nous voudrions comparer les modèles de résolution de problèmes préexistants avec les résultats de nos études pour concrétiser un nouveau modèle. Entre autres, nos résultats nous permettront de créer un modèle concret du phénomène complexe de la résolution de problèmes dans les laboratoires de fabrication numérique avec tinkering au cœur du processus. 


\section{REVUE HYBRIDE DE L'ÉDUCATION}

\section{Références}

Bevan, B., Gutwill, J.P., Petrich, M. et Wilkinson, K. (2015). Learning through STEM-rich tinkering: Findings from a jointly negotiated research project taken up in practice. Science Education, 99(1), 98-120. https://doi.org/10.1002/sce.21151

Bevan, B., Petrich, M. et Wilkinson, K. (2014). Tinkering is serious play. Educational Leadership, 72(4), 28-33. https://www.researchgate.net/profile/Bronwyn Bevan/publication/ 289611225 Tinkering is serious play/links/5757ea1d08ae5c654 907263b/Tinkering-is-serious-play.pdf

Blikstein, P. (2013). Digital fabrication and 'making' in education: the democratization of invention. Dans J. W.-H. C. Büching (dir.), FabLabs : Of Machines, Makers and Inventors. Transcript Publishers.

Cross, A. (2017). Tinkering in k-12: an exploratory mixed methods study of makerspaces in schools as an application of constructivist learning [thèse de doctorat, Pepperdine University]. ProQuest Dissertations and Theses Global. Publication no 10265494

Dede, C. (2010). Comparing frameworks for the 21st century skills. Dans J.A. Bellanca et R.S. Brandt (dir.), 21st century skills : Rethinking how students learn (p. 51-76). Solution Tree Press.

Doorley, R. (2015). What is Tinkering? Tinkerlab. http:// tinkerlab.com/what-is-tinkering/

Economist Intelligence Unit. (2015). Driving the skills agenda: Preparing students for the future. London: The Economist Intelligence Unit Limited.

https://eiuperspectives.economist.com/sites/default/files/Drivingth eskillsagenda.pdf

Furlong, C., Léger, M.T. et Freiman, V. (2019). Le développement de compétences numériques lors de fabrication numérique : cas de Labos créatifs. La Revue canadienne de l'apprentissage et de la technologie, 45(2), 1-23. https://doi.org/10.21432/cj|t27831

Gauthier, M. (2014). Perceptions des élèves du secondaire par rapport à la résolution de problèmes en algèbre à l'aide d'un logiciel dynamique et la stratégie Prédire-investiguer-expliquer. Education et francophonie, 42(2), 190-214. https://doi.org/10.7202/1027913ar 


\section{8}

\section{REVUE HYBRIDE DE L'ÉDUCATION}

Gewertz, C. (2007). Soft skills in big demand. Education Week, 26(40), 2527.

https://www.edweek.org/ew/articles/2007/06/12/40soft.h26.html

Griffin, P., McGaw, B. et Care, E. (2015). Assessment and teaching of 21st century skills. Springer.

Groff, J. (2013). Technology-rich innovative learning environments. OCED CERI Innovative Learning Environment project, 1-30. https://www.researchgate.net/profile/Jennifer Groff/publication/30 7981656 Technologyrich innovative learning environments/links/5b1686d1a6fdcc31b bf5a7c6/Technology-rich-innovative-learning-environments.pdf

Hancock, C. (2003). Real-time programming and the big ideas of computational literacy [thèse de doctorat, Massachusetts Institute of Technology]. MIT Media Lab. http://citeseerx.ist.psu.edu/viewdoc/download?doi=10.1.1.94. $1714 \&$ rep=rep $1 \&$ type $=$ pdf

Harris, E., Winterbottom, M., Xanthoudaki, M. et InKa de Pijer, C. (2016). Tinkering: A Practitioner Guide for Developing and Implementing Tinkering Activities. Tinkering: Contemporary Education for Innovators of Tomorrow Project. https://www.researchgate.net/publication/306066132 A PRACTI TIONER GUIDE FOR DEVELOPING AND IMPLEMENTING T INKERING ACTIVITIES

Hesse, F., Care, E., Buder, J., Sassenberg, K. et Griffin, P. (2015). A framework for teachable collaborative problem solving skills. Dans P. Griffin, B. et E. Care (dir.), Assessment and teaching of 21st century skills: Methods and approaches (p. 37-56). Springer.

Kim, M.C. et Hannafin, M.J. (2011). Scaffolding problem solving in technology-enhanced learning environments (TELEs): Bridging research and theory with practice. Computers \& Education, 56(2), 403-417. https://doi.org/10.1016/i.compedu.2010.08.024

Laperrière, A. (1997). Les critères de scientificité des méthodes qualitatives. Dans J. Poupart, J.P. Deslauriers, L. Groulx, A. Laperrière, R. Mayer et A. Pirès (dir.), La recherche qualitative, enjeux épistémologiques et méthodologiques (p. 365-389). Gaétan Morin Éditeur.

LeBlanc, M., Freiman, V. et Furlong, C. (2018). STEAMing soft-skills in makerspaces: What are the mathematical connections? Dans $C$. Michelsen, A. Beckmann, V. Freiman et U.T. Jankvist (dir.), Actes 


\section{REVUE HYBRIDE DE L'ÉDUCATION}

de I'International Symposium MACAS (p. 87-97) Laboratorium for Sammenhængende Undervisnings og Læring (LSUL).

Lecompte, M. D. et Preiss, J. G. (1993). Ethnography and Qualitative Design in Educational Research (2e éd.). Emerald group publishing.

Martinez, S. L. et Stager, G. (2013). Invent to learn: Making, tinkering, and engineering in the classroom. Constructing Modern Knowledge Press.

Ministère de l'Éducation et de l'Enseignement supérieur. (2019). Cadre de référence de la compétence numérique. Gouvernement du Québec.

http://www.education.gouv.qc.ca/fileadmin/site web/documents/m inistere/Cadre-reference-competence-num.pdf

Ministère de l'Éducation et du Développement de la petite enfance. (2018).

Programme d'études - Mathématiques 30131 (9e année). Gouvernement du Nouveau-Brunswick. http://142.139.25.169/content/dam/gnb/Departments/ed/pdf/K12/s ervped/Mathematiques/Mathematiques-7eAnnee.pdf

Organisation de cooperation et de développement économiques (OCDE). (2012). Literacy, Numeracy and Problem solving in TechnologyRich Environments: Frameworks for the OECD Survey of Adult Skills. OECD Publishing.

Paillé, P. et Mucchielli, A. (2008). L'analyse qualitative en sciences humaines et sociales ( $2^{\mathrm{e}}$ éd.). Armand Colin.

Parekh, P. et Gee, E. R. (2018). Zooming into a Tinkering Project: The Progression of Learning through Transitional Objects. Interdisciplinary Journal of Problem-Based Learning, 12(2). https://doi.org/10.7771/1541-5015.1751

Peppler, K., Maltese, A., Keune, A., Chang, S. et Regalla, L. (2015). The maker ed open portfolio project: Survey of Makerspaces, Part II. Dans S. Chang, A. Keune, K. Peppler et L. Regalla (dir.), Open Portfolio Project Research Brief Series (p.47-53). Maker Education Initiative.

Petrich, M., Wilkinson, K., et Bevan, B. (2013). It looks like fun, but are they learning? Dans M. Honey et D. Kanter, (dir.), Design, make, play : Growing the next generation of STEM innovators (p.5070). Routledge. 


\section{REVUE HYBRIDE DE L'ÉDUCATION}

Poce, A., Amenduni, F. et De Medio, C. (2019). From Tinkering to Thinkering. Tinkering as Critical and Creative Thinking Enhancer. Journal of e-Learning and Knowledge Society, 15(2). https://doi.org/10.20368/1971-8829/1639

Poirier, L. (2001). Enseigner les maths au primaire [Notes didactiques]. Montréal : ERPI

Resnick, M. et Rosenbaum, E. (2013). Designing for Tinkerability. Dans M. Honey et D. E. Kanter (dir.), Design, Make, Play : Growing the Next Generation of STEM Innovators (p. 163-181). New Routledge.

Rosa, P., Ferretti, F., Pereira, Â.G., Panella, F. et Wanner, M. (2017). Overview of the maker movement in the European Union. Publications Office of the European Union.

Savoie-Zajc, L. (2018). La recherche qualitative/interprétative en éducation. Dans T. Karsenti et L. Savoie-Zajc (dir.). Introduction à la recherche en éducation ( $4^{\mathrm{e}}$ éd., p. 101-217). Éditions du CRP.

Schleicher, A. (2018). World Class: How to build a 21st-century school system. OECD Publishing.

Siddiq, F., Gochyyev, P. et Wilson, M. (2017). Learning in Digital NetworksICT literacy: A novel assessment of students' 21st century skills. Computers \& Education, 109, 11-37. http://dx.doi.org/10.1016/i.compedu.2017.01.014

Smith, R. C., Iversen, O. S. et Hjorth, M. (2015). Design thinking for digital fabrication in education. International Journal of Child-Computer Interaction, 5, 20-28. http://dx.doi.org/10.1016/j.ijcci.2015.10.002

Statistique Canada. (2013). Skills in Canada: First Results from the Program for the International Assessment of Adult Competencies (PIAAC).

Publication

no $89-555-X$

https://www.statcan.gc.ca/pub/89-555-x/89-555-x2013001eng.htm

Van Holm, E. (2014, 7 novembre). What are makerspaces, hackerspaces, and fab labs?, SSRN Electronic Journal http://dx.doi.org/10.2139/ssrn.2548211

Voogt, J. et Roblin, N.P. (2012). A comparative analysis of international frameworks for 21st century competences: Implications for national curriculum policies. Journal of Curriculum Studies, 44(3), 299-232. http://dx.doi.org/10.1080/00220272.2012.668938. 


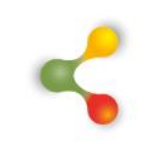

\section{REVUE HYBRIDE DE L’ÉDUCATION}

Vossoughi, S. et Bevan, B. (2014). Making and Tinkering: A review of the literature. National Research Council Committee on Successful Out-of-School STEM Learning. National Research Council.

Vossoughi, S., Hooper, P.K. et Escudé, M. (2016). Making through the lens of culture and power: Toward transformative visions for educational equity. Harvard Educational Review, 86(2), 206-232. https://www.sesp.northwestern.edu/docs/publications/718280483 5761a089d5180.pdf

Wilkinson, K. et Petrich, M. (2014). The Art of Tinkering: Meet 150 Makers Working at the Intersection of Art, Science \& Technology. Weldon Owen.

Wilson, M., Scalise, K., et Gochyyev, P. (2015). Rethinking ICT literacy: From computer skills to social network settings. Thinking Skills and Creativity, 18, 65-80. http://dx.doi.org/10.1016/j.tsc.2015.05.001 